\title{
Prevalence of Multidrug Resistant Enterococci in a Tertiary Care Hospital in India: A Growing Threat
}

\author{
Nita Gangurde ${ }^{1}$, Manisha Mane ${ }^{2}$, Sunita Phatale ${ }^{1}$ \\ ${ }^{1}$ Dr. V. P. Medical College, Hospital \& RC, Nashik, India \\ ${ }^{2}$ ESIC-Medical College, PGIMSR, Chennai, India \\ Email: nitagangurde@gmail.com
}

Received 11 December 2013; revised 10 January 2014; accepted 17 January 2014

Copyright (C) 2014 by authors and Scientific Research Publishing Inc.

This work is licensed under the Creative Commons Attribution International License (CC BY). http://creativecommons.org/licenses/by/4.0/ (c) (i) Open Access

\begin{abstract}
Introduction: Enterococci are members of the healthy human intestinal flora, but are also leading causes of highly antibiotic-resistant infections. Serious enterococcal infections are often difficult to treat since the organisms have a tremendous capacity to acquire resistance to penicillin, high concentration of aminoglycoside \& vancomycin. Careful review of in vitro susceptibility data is required to treat infections caused by MDR Enterococci. Therefore we conducted the study to find out prevalence of MDR Enterococci. Aims \& Objectives: To study the prevalence of Vancomycin resistance, High Level Streptomycin Resistance (HLSR) \& High Level Gentamicin Resistance (HLGR) in different enterococcal isolates. Materials \& Methods: Total 180 enterococcal isolates were studied. Identification was done by conventional biochemical methods. Antibiotic susceptibility testing was done by Kirby-Bauer disc diffusion method on Mueller-Hinton agar and results were interpreted as per CLSI guidelines. HLSR \& HLGR was determined by disc diffusion method using high level Gentamicin disc (120 $\mu \mathrm{g})$ \& Streptomycin $(300 \mu \mathrm{g})$ discs. Minimum inhibitory concentration (MIC) determination for Vancomycin was done by vancomycin E test strips. Results: Total 180 entetococcal isolates were studied. E. faecalis was $60 \%$, E. faecium was $32.2 \%$, E. durans and $E$. raffinosus were $4.4 \%$ \& $3.3 \%$ respectively. Enterococcus fecium showed resistance in high percentage as compared to E. faecalis. 15 isolates were found to be vancomycin resistant. Conclusion: Resistance to aminoglycoside is of great concern. Regular screening of enterococcal isolates for vancomycin resistance detection should be implemented. It is very important to implement infection control measures, screening of health care workers, surveillance cultures in intensive care units which can control spread of multidrug resistant enterococci.
\end{abstract}

\section{Keywords}

Enterococci; HLGR; HLSR; VRE

How to cite this paper: Gangurde, N., Mane, M. and Phatale, S. (2014) Prevalence of Multidrug Resistant Enterococci in a Tertiary Care Hospital in India: A Growing Threat. Open Journal of Medical Microbiology, 4, 11-15. 


\section{Introduction}

Enterococci are members of the healthy human intestinal flora, but are also leading causes of highly antibioticresistant, hospital-acquired infection [1]. There is growing evidence that these bacteria frequently possess several specific traits that enable them to survive in the hospital environment, colonize patients, and cause infections such as bacteraemia, peritonitis, endocarditis and urinary tract, wound, and device-related infections [2]. Serious enterococcal infections are often difficult to treat since the organisms have a tremendous capacity to acquire resistance to penicillin, high concentration of aminoglycoside \& vancomycin [3]. Enterococci with high level resistance to aminoglycosides (HLAR), beta lactamase production \& glycopeptide resistance including vancomycin resistance are posing a great therapeutic challenge, not only for clinicians but also for healthcare institutions [4]. Multidrug resistance complicates treatment of enterococcal infections and the therapeutic spectrum of these cases is limited. Careful review of in vitro susceptibility data is required to treat infections caused by MDR E. faecium, the most commonly found group of VRE. Empiric therapy of enterococcal infections should be guided by local patterns of drug resistance. Nowadays emergence of MDR enterococci is thought to be due to antibiotic selective pressure. This organism is considered as second leading cause of hospital acquired infections [5]-[7]. Therefore we conducted the study to find out prevalence of drug resistance in Enterococcal isolates with regards to HLAR (HLSR \& HLGR) and Vancomycin resistance in our set up.

\section{Aims \& Objectives}

To study the prevalence of

1) Vancomycin resistance in different Enterococcal isolates;

2) High Level Streptomycin Resistance (HLSR) \& High Level Gentamicin Resistance (HLGR) in different enterococcal isolates.

\section{Materials \& Methods}

Total 180 enterococcal isolates obtained from clinical samples received in the Department of Microbiology, Dr. Vasantrao Pawar Medical College, Hospital \& Research Centre, Nashik, India. The clinical specimens were received for routine culture and sensitivity during August 2012 to July 2013 was included in the study.

The isolates were identified by colony morphology, Gram's staining, catalase production, growth in nutrient broth containing $6.5 \% \mathrm{NaCl}$, aesculin hydrolysis in presence of $40 \%$ bile salts, growth at $10^{\circ} \mathrm{C}, 37^{\circ} \mathrm{C} \& 45^{\circ} \mathrm{C} \&$ other biochemical reactions [6] [8] [9]. Antibiotic susceptibility testing for ampicillin, amoxyclav, chloramphenicol, erythromycin, cotrimoxazole, ciprofloxacin, teicoplanin was done by Kirby-Bauer disc diffusion method [10] [11] on Mueller-Hinton agar and results were interpreted as per CLSI guidelines [6] [12] [13]. Enterococcus feacalis ATCC 29212 [Hi Media Laboratories, Mumbai] was used as quality control strain. HLSR \& HLGR was determined by disc diffusion method using high level Gentamicin disc $(120 \mu \mathrm{g}) \&$ Streptomycin $(300 \mu \mathrm{g})$ discs [Hi Media Laboratories, Mumbai]. A diameter of the zone of inhibition $<6 \mathrm{~mm}$ indicated resistance, 7 - 9 $\mathrm{mm}$ as intermediate and $\geq 10 \mathrm{~mm}$ as susceptible [10] [14].

Screening for Vancomycin resistance was performed by using Vancomycin screen agar [BHI agar with 6 $\mu \mathrm{g} / \mathrm{ml}$ Vancomycin] (Hi-media Laboratory, Mumbai). One or more colony indicated resistance to Vancomycin. Control used were E. faecalis ATCC 51299 [Hi-media Laboratory, Mumbai] as positive control and E. faecalis ATCC 29212 (Hi-media Laboratory, Mumbai) as negative control. Minimum inhibitory concentration (MIC) determination was done by Vancomycin E test strips (Hi-media Laboratory, Mumbai). Since E test is convenient to perform, MIC determination of Vancomycin against VRE were done by E test (Hi-media Laboratory, Mumbai) [6] [15] [16]. Test procedure was performed as per the manufacturer's instructions. MIC values $\leq 4 \mu \mathrm{g} / \mathrm{ml}$ was taken as susceptible and $\geq 32 \mu \mathrm{g} / \mathrm{ml}$ as resistant [6] [15] [16]. Enterococcus faecalis ATCC 29212 and $S$. aureus ATCC 25923 were used as control strains [6] [17].

\section{Results}

Total 180 enterococcalisolates obtained from clinical samples over a period of one year were studied. We found E. faecalis as commonest (60\%) species. E. faecium was $32.2 \%$ and other enterococci included E. durans $(\mathrm{n}=8)$ and E. raffinosus $(\mathrm{n}=6)$ (Table 1$)$. 
Enterococcus faecium showed resistance in high percentage as compared to E. faecalis. Total 63 enterococcal isolates showed High level streptomycin resistance (HLSR). Out of those 63 isolates 23 were E. faecalis, 34 were $E$. faecium and other enterococci were 6 in numbers. Also High level Gentamicin resistance (HLGR) was shown by 54 enterococcal isolates. Out of which 21 isolates were E. faecalis, 29 were E. faecium and 4 were other enterococci. 15 isolates were found to be Vancomycin resistant, of which 4 had MIC between $8-16 \mu \mathrm{g} / \mathrm{ml}$. VRE included 5 isolates of $E$. faecalis, 8 isolates of E. faecium, one isolate of $E$. durans and one isolate of $E$. raffinosus. all isolates were sensitive to teicoplanin (Table 2).

\section{Discussion}

In our study, E. faecalis was the predominant species. This findings of our study are similar with findings of S. Farnandes et al. and S. Bose et al. [6] [10]. But there are few studies from north India (S. Jain et al.) [18], Mumbai (Karmarkar et al.) [7] and South India (Telkar et al.) [14] found E. faecium as predominant species. Also our these findings for E. faecalis as predominant species are similar to other studies from different parts of India [4] [17] [19] [20].

Enterococci are widely distributed in nature and are usually part of mixed flora commonly found in gastrointestinal tract and remains difficult to differentiate colonization from true infection [19]. Intensive use of broad spectrum antibiotics is responsible for conversion of enterococci to opportunistic nosocomial pathogens (Huycke et al., 1998) [1], Mohanty et al., 2005) [3]. Enterococci demonstrate both intrinsic as well as extrinsic types of resistance to antibiotics [6]. Our study revealed presence of multidrug resistant enterococcal species. Our findings are similar with other studies (Mathur et al., A. Telkar et al., Senal et al.) [10] [14] [21]. In our study we found $E$. faecium to be more drug resistant than $E$. faecalis. similar findings have been reported by other studies [10] [14] [18]. Resistance to ampicillin was found to be higher in E. faecium in our study as compared to E. faecalis. Similar findings have been reported by Kalyan et al. [22]. Erythromycin resistance in our study was quite high in accordance with findings reported by Sanal et al., Mathur et al., Agarwal J et al. [10] [21] [23]. Also almost 51\% isolates were resistant to chloramphenicol. Cotrimoxazole resistance was almost equal in both the species. Frequent use of these antimicrobials for empirical treatment of enterococcal infections could be

Table 1. Number and percentage (\%) of different species of Enterococci isolated in our study.

\begin{tabular}{cccc}
\hline E. faecalis & E. faecium & E. durans & E. raffinosus \\
$\mathrm{n}=108$ & $\mathrm{n}=58$ & $\mathrm{n}=8$ & $\mathrm{n}=6$ \\
$60 \%$ & $32.2 \%$ & $4.4 \%$ & $3.3 \%$ \\
\hline
\end{tabular}

\begin{tabular}{ccccc}
\multicolumn{5}{c}{ Table 2. Antibiotic resistance (\%) among enterococcal isolates. } \\
\hline ANTIBIOTIC & $\begin{array}{c}\text { E. faecalis \% } \\
(\boldsymbol{n}=\mathbf{1 0 8})\end{array}$ & $\begin{array}{c}\text { E.faecium } \% \\
(\boldsymbol{n}=\mathbf{5 8})\end{array}$ & $\begin{array}{c}\text { E. durans \% } \\
(\boldsymbol{n}=\mathbf{8})\end{array}$ & $\begin{array}{c}\text { E. raffinosus \% } \\
(\boldsymbol{n}=\mathbf{6})\end{array}$ \\
\hline Ampicillin & $41.6(45)$ & $55.1(32)$ & $37.5(3)$ & $33.5(2)$ \\
Amoxyclav & $81.4(88)$ & $81.0(47)$ & $75(6)$ & $66.6(4)$ \\
Erythromycin & $85.1(92)$ & $91.3(53)$ & $25(2)$ & $33.5(2)$ \\
Chloramphenicol & $50.9(55)$ & $48.2(28)$ & $37.5(3)$ & 0 \\
Cotrimoxazole & $70.3(76)$ & $72.4(42)$ & 0 & 0 \\
Ciprofloxacin & $80.5(87)$ & $86.2(50)$ & $87.5(7)$ & $83.3(5)$ \\
HLSR & $42.5(46)$ & $55.1(32)$ & $12.5(1)$ & $33.3(2)$ \\
HLGR & $44.4(48)$ & $53.4(31)$ & $12.5(1)$ & $50(2)$ \\
Teicoplanin & 0 & 0 & 0 & 0 \\
Vancomycin & $4.6(5)$ & $13.7(8)$ & $12.5(1)$ & $16.6(1)$ \\
\hline
\end{tabular}


the reason for this antibiotic resistance. All the isolates were sensitive to teicoplanin. Therefore indicating teicoplanin as a reserve drug for therapy against these notorious organisms. These findings are in congruence with other similar studies [20].

According to our study High level Gentamicin resistance (HLGR) was $44.4 \%$ in E. faecalis and $55.1 \%$ in $E$. faecium. We found HLGR in higher percentage in E. faecium than in E. faecalis. This finding matches with some studies. Sanal Farnandes et al. have reported similar findings. Also study by Loveena et al. [4] reports HLGR higher in E. faecium than E. faecalis. A Telkar et al. reports HLGR of $71.4 \%$ in E. faecium and $57 \%$ in $E$. faecalis. Also Mendiratta et al. have reported greater resistance to HLG among E. faecium as compared to E. faecalis. Studies from New Delhi and Mumbai have reported HLGR prevalence to be as high as $70 \%$ and $100 \%$ respectively [7] [24]. High level streptomycin resistance was $42.5 \%$ in E. faecalis and $55.1 \%$ in E. faecium. Our this finding of HLSR being greater in E. faecium than E. faecalis is in accordance with other studies (Sanal et al., Loveena et al., Telkar A et al., Sarika Jain et al.). But Ramalingam S et al. [25] found HLSR higher in E. faecalis than E. faecium.

The emergence of Vancomycin resistant enterococci poses a serious threat to hospitalized patients with impaired host defenses [3]. In India, the prevalence of VRE has been reported to be between 0 - 30 percent [21] [23] [26]-[28]. In our study, 15 isolates were found to be resistant to Vancomycin with E. faecium (13.7\%) showing higher resistance than E. faecalis (4.6\%). Similar studies by Sanal et al., Telkar et al. \& Karmarkar et $a l$. had also reported greater resistance among E. faecium isolates [7] [10] [14]. Agarwal et al. found Vancomycin resistance to be greater among $E$. faecalis isolates. Thus the present study indicated an increase in Vancomycin resistance of the enterococcal isolates. The Vancomycin resistance in enterococci not only leaves fewer options for the disease management, but it is also important due to the potential risk of the Vancomycin resistance gene transfer from the enterococci to Staphylococcus aureus [29]. Also emergence of Vancomycin resistant enterococci has been attributed to imprudent use of cephalosporins, Vancomycin, colonization pressure and noncompliance with infection control measures [7]. All the VRE isolates in our study found to be sensitive to teicoplanin, leaving teicoplanin as treatment options.

\section{Conclusions}

Resistance to aminoglycoside is of great concern, since it eliminates the synergy of aminoglycosides with beta lactam antibiotics, which is the therapy of choice for most of enterococcal infection which can limit the therapeutic options. Also now a day there is emergence of Vancomycin resistance, therefore regular screening of enterococcal isolates for Vancomycin resistance detection should be implemented to limit the spread of MDR enterococci.

Also since enterococci can survive in hospital environment due to their intrinsic resistance to several commonly used antibiotics and their ability to acquire resistance to all currently available antibiotics by mutation or through plasmid, it is very important to implement infection control measures, screening of health care workers, surveillance cultures in intensive care units which can control spread of multidrug resistant enterococci.

\section{References}

[1] Shankar, N., Baghdayan, A.S. and Gilmore, M.S. (2002) Modulation of Virulence within a Pathogenicity Island in Vancomycin-Resistant Enterococcus faecalis. Nature, 417, 746-750. http://dx.doi.org/10.1038/nature00802

[2] Sava, G., Heikens, E. and Huebner, J. (2010) Pathogenesis and Immunity in Enterococcal Infections. Clinical Microbiology and Infection, 16, 533-540. http://dx.doi.org/10.1111/j.1469-0691.2010.03213.x

[3] Mohanty, S., Dhawan, B., Gadepalli, R.S., Lodha, R. and Kapil, A. (2006) Case Report of Vancomycin Resistant Enterococcus Faecium VanA Phenotype: First Documented Isolation in India. The Southeast Asian Journal of Tropical Medicine and Public Health, 37, 335-337.

[4] Oberoi, L., et al. (2010) Multidrug Resistant Enterococci in a Rural Tertiary Care Hospital—A Cause of Concern. JK Science, 12, 157-158.

[5] Fraser, S.L. (2011) Enterococcal Infection. Medscape, Drugs, Diseases and Procedures. http://emedicine.medscape.com/article/216993-overview

[6] Bose, S., Ghosh, A. and Barapatre, R. (2012) Prevalence of Drug Resistance among Enterococcus Species Isolated from A Tertiary Care Hospital. International Journal of Medical Health Sciences, 1, 38-44.

[7] Karmarkar, M.G., Gershom, E.S. and Mehta, P.R. (2004) Enterococcal Infections with Special Reference to Phenotypic 
Characterization \& Drug Resistance. Indian Journal of Medical Research, 119, 22-25.

[8] Forbes, A.B., Sahm, D.F. and Weissfeld, A.S. (2007) Chapter 13: Overview of Bacterial Identification Methods and Strategies. In: Baily \& Scott’s Diagnostic Microbiology 12th Ed., Mosby Elsevier International Edition, 216-247.

[9] Collee, J.G., Fraser, A.G., Marmion, B.P. and Simmons, A. (2008) Chapter 7: Test for Identification of Bacteria. In: Collee, J.G., Miles, R.S. and Watt, B., Eds., Test for Identification of Bacteria, Mackie \& McCartney's Practical Medical Microbiology, 14th Edition, Indian Reprint, Churchil Livingstone, 131-149.

[10] Fernandes, S.C., Dhanashree, B., et al. (2013) Drug Resistance \& Virulence Determinants in Clinical Isolates of Enterococcus Species. Indian Journal of Medical Research, 137, 981-985.

[11] Bauer, A.W., Kirby, W.M., Sherris, J.C. and Turck, M. (1966) Antibiotic Susceptibility Testing by a Standardized Single Disk Method. American Journal of Clinical Pathology, 45, 493-496.

[12] McNamara, E.B., King, E.M. and Smyth, E.G. (1995) A Survey of Antimicrobial Susceptibility of Clinical Isolates of Enterococcus Spp. from Irish Hospitals. Journal of Antimicrobial Chemotherapy, 35, 185-189. http://dx.doi.org/10.1093/jac/35.1.185

[13] Wayne, P.A. (2010) Clinical \& Laboratory Standard Institute, Performance Standard for Antimicrobial Susceptibility Testing. Twentieth Informational Supplement, 30, M100-M120.

[14] Telkar, A., et al. (2012) Change in the Prevalence and the Antibiotic Resistance of the Enterococcal Species Isolated from Blood Culture. Journal of Clinical and Diagnostic Research, 6, 405-407.

[15] Yemisen, M., Demirel, A., Mete, B., Kaygusuz, A., Mert, A., Tabak, F. and Ozturk, R. (2009) Comparative in Vitro Antimicrobial Activity of Tigecycline against Clinical Isolates of Vancomycin-Resistant Enterococcus. Indian Journal of Medical Microbiology, 27, 373-374. http://dx.doi.org/10.4103/0255-0857.55455

[16] De, A., Bindlish, A., Kumar, S. and Mathur, M. (2009) Vancomycin Resistant Enterococci in a Tertiary Care Hospital in Mumbai. Indian Journal of Medical Microbiology, 27, 375-376. http://dx.doi.org/10.4103/0255-0857.55451

[17] Mendiratta, D.K., Kaur, H., Deotale, V., Thamke, D.C., Narang, R. and Narang, P. (2008) Status of High Level Aminoglycoside Resistant Enterococcus Faecium and Enterococcus Faecalis in a Rural Hospital of Central India. Indian Journal of Medical Microbiology, 26, 369-371. http://dx.doi.org/10.4103/0255-0857.43582

[18] Jain, S., Kumar, A., Kashyap, B. and Kaur, I.R. (2011) Clinico-Epidemiological Profile and High-Level Aminoglycoside Resistance in Enterococcal Septicemia from a Tertiary Care Hospital in East Delhi. International Journal of Applied and Basic Medical Research, 1, 80-83. http://dx.doi.org/10.4103/2229-516X.91149

[19] Chaudhari, U., et al. (2007) Antimicrobial Susceptibility Pattern of Common and Unusual Enterococcus Species Isolated from Clinical Specimens. Journal of Infectious Diseases and Antimicrobial Agents, 24, 55-62.

[20] Shah, L., et al. (2012) Prevalence of Enterococci with Higher Resistance Level in a Tertiary Care Hospital: A Matter of Concern. National Journal of Medical Research, 2, 25-27.

[21] Mathur, P., Kapil, A., Chandra, R., Sharma, P. and Das, B. (2003) Antimicrobial Resistance in Enterococcus Faecalis at a Tertiary Care Centre of Northern India. Indian Journal of Medical Research, 118, 25-28.

[22] Kalyan, R., et al. (2013) Vancomycin Resistant Enterococci at a Tertiary Care Hospital in Northern India. International Journal of Pharma and Bio Sciences, 4, 1090-1094.

[23] Agarwal, J., Kalyan, R. and Singh, M. (2009) High-Level Aminoglycoside Resistance and Beta-Lactamase Production in Enterococci at a Tertiary Care Hospital in India. Japanese Journal of Infectious Diseases, 62, 158-159.

[24] Randhawa, V.S., Kapoor, L., Singh, V. and Mehta, G. (2004) Aminoglycoside Resistance in Enterococci Isolated from Paediatric Septicaemia in a Tertiary Care Hospital in North India. Indian Journal of Medical Research, 119, 77-79.

[25] Sekar, R., Srivani, R., Vignesh, R., Kownhar, H. and Shankar, E.M. (2008) Low Recovery Rates of High-Level Aminoglycoside-Resistant Enterococci Could be Attributable to Restricted Usage of Aminoglycosides in Indian Settings. Journal of Medical Microbiology, 57, 397-398. http://dx.doi.org/10.1099/jmm.0.47604-0

[26] Kapoor, L., Randhawa, V.S. and Deb, M. (2005) Antimicrobial Resistance of Enterococcal Blood Isolates at a Pediatric Care Hospital in India. Japanese Journal of Infectious Diseases, 58, 101-103.

[27] Taneja, N., Rani, P., Emmanuel, R. and Sharma, M. (2004) Significance of Vancomycin Resistant Enterococci from Urinary Specimens at a Tertiary Care Centre in Northern India. Indian Journal of Medical Research, 119, 72-74.

[28] Gupta, V. and Singla, N. (2007) Antibiotic Susceptibility Pattern of Enterococcai. Journal of Clinical and Diagnostic Research, 5, 385-389.

[29] Huycke, M.M., Sahm, D.F. and Gilmore, M.S. (1998) Multiple-Drug Resistant Enterococci: The Nature of the Problem and an Agenda for the Future. Emerging Infectious Diseases, 4, 239-249. http://dx.doi.org/10.3201/eid0402.980211 